\title{
ASSISTÊNCIA À SAÚDE MENTAL VOLTADA PARA MULHERES NA ATENÇÃO PRIMÁRIA
}

\author{
Luana Adrielle Leal Dantas I \\ Lorrainy da Cruz Solano"
}

Lucídio Clebeson de Oliveira III

\begin{abstract}
RESUMO
A atenção básica é classificada como a principal forma de acesso do usuário ao Sistema Único de Saúde, proporcionando ações coletivas e individuais, visando a promoção e proteção à saúde, bem como o diagnóstico precoce, adesão ao tratamento e reabilitação, promovendo a melhoria do bem-estar das coletividades. Objetiva-se realizar uma revisão de literatura acerca da presença da população feminina no serviço de atenção básica, proveniente de queixas relacionadas à saúde mental. Trata-se de um levantamento bibliográfico, realizado no período de 2011 a 2018, nas bases de dados Lilacs, Scielo e Medline. Foram utilizados os descritores: assistência à saúde, transtornos mentais e saúde da mulher. Como critérios de inclusão adotou-se: artigos publicados no período de 2011 a 2018, que tenham consistência metodológica e que versem sobre a temática, sendo excluídos os que não atendessem aos critérios citados. Foram encontrados na pré-análise 146 artigos. No entanto, após a leitura dos títulos e resumos, foram excluídos os que não atenderam a temática proposta, sendo selecionados 20 artigos e destes, somente 16 atenderam aos critérios de inclusão. A análise de dados foi realizada através da avaliação temática de conteúdo, proposta por Bardin. Por meio da leitura dos artigos, foram identificadas três categorias: violência moral, sexual e doméstica, transtornos mentais mais prevalentes e uso de álcool e outras drogas. Foi evidenciado que a violência psicológica tem maior prevalência, seguida pela violência física. Como fatores determinantes destacam-se o baixo nível de escolaridade, a situação conjugal, histórico materno de violência por parceiro íntimo e uso de drogas. Por fim, a violência sexual, tida como o terceiro tipo mais predominante, associada a renda familiar, predispondo o desenvolvimento dos transtornos mentais comuns, além do uso abusivo de psicofármacos, estresse, ansiedade e problemas conjugais. Neste sentido, entende-se que as mulheres em sofrimento mental, têm inúmeras razões que desencadeiam seu estado de saúde, estando a violência e o abuso no consumo de álcool e outras drogas, dentre os fatores mais relacionados a etiologia dos transtornos mentais.
\end{abstract}

PALAVRAS-CHAVE: Assistência à Saúde. Transtornos Mentais. Saúde da Mulher.

Acadêmica de Enfermagem, Faculdade de Enfermagem Nova Esperança, Grupo de Estudo e Pesquisa em Ciências da Saúde da FACENE, Mossoró, Rio Grande do Norte, Brasil. Autora correspondente: Email:Iuanadantas904@gmail.com. ORCID: 0000-0001-6747-3513

Enfermeira. Mestre e Doutoranda em Enfermagem. Docente da Universidade do Estado Rio Grande do Norte, Mossoró, Rio Grande do Norte, Brasil. ORCID ID: 0000000-0002-4426-7618

Enfermeiro. Mestre em enfermagem e doutorando em Psicobiologia - Universidade Federal do Rio III Grande do Norte (UFRN). Docente da Universidade do Estado do Rio Grande do Norte. Mossoró, Rio Grande do Norte, Brasil. ORCID ID: 0000-0002-2033-7546 


\section{INTRODUÇÃO}

A atenção básica é classificada como a principal forma de acesso do usuário ao Sistema Único de Saúde, proporcionando ações coletivas e individuais, visando a prevenção, promoção e proteção à saúde, bem como o diagnóstico precoce, adesão ao tratamento e reabilitação, com o intuito de promover a meIhoria do bem-estar das coletividades. Por meio da aprovação da lei $n^{\circ} 10.216$, foi sancionado o direito das pessoas portadoras de transtornos mentais, também orientou o modelo assistencial de saúde mental, com a institucionalização do decreto presidencial $n^{\circ} 7508 / 2011$, que substituiu o paradigma manicomial e os serviços de saúde como: os Centros de Atenção Psicossocial (CAPS), os Serviços Residenciais Terapêuticos (SRT), os Centros de Convivência (Cecos), as Enfermarias de Saúde e as Unidades Básicas de Saúde. ${ }^{1}$

A atenção primária é composta pelos princípios da integralidade e envolvimento social que funcionam como forma de interação entre a população e as práticas de saúde mental, bem como contribui para a compreensão do conceito de saúde-doença e do modelo psicossocial. Essa nova perspectiva de atenção está sendo implementada visando a substituição do modelo hospitalocêntrico, centralizando o cuidado social e familiar. ${ }^{2}$

Já na rede de atenção básica, as unida-

\section{MATERIAL E MÉTODOS}

Esse trabalho foi elaborado por meio de uma revisão sistemática nas principais bases de dados, Lilacs, Scielo e Medline, no período de 2011 e 2019. Foram utilizados como des de saúde são a porta de entrada da população ao serviço público de saúde. Nesta rede, diariamente, diversas queixas são relatadas pelos usuários, sendo as principais, insônia, nervosismo, dores de cabeça, fadiga, dores somáticas falta de concentração e o aparecimento de sintomas depressivos. 3

Ao analisar a saúde mental no Brasil, percebe-se que o maior público acometido é o feminino, composto por mulheres residentes em áreas urbanas, com baixo nível escolar e doenças crônicas como hipertensão e diabetes. 4

Além da predisposição das mulheres a adquirirem os transtornos mentais comuns (TMC), o fato de o público feminino ter uma maior assiduidade aos serviços de saúde, torna-se mais acessível e fácil a adesão dessa população a ações de promoção da saúde, bem como de prevenção e tratamento à doenças e agravos.3 Nessa perspectiva, emergem as questões de pesquisa desse estudo: Quais as principais razões das mulheres procurarem a atenção básica de saúde, em busca de assistência à saúde mental?

Dessa forma, a presente pesquisa objetiva realizar uma revisão de literatura acerca da presença da população feminina no serviço de atenção básica, proveniente de queixas relacionadas à saúde mental. 
separadamente. Em seguida, pesquisados combinados, interligados através do operador boleano "AND". Como critérios de inclusão adotou-se: artigos publicados no período de 2011 a 2018, que apresentam consistência metodológica e que versam sobre a temática, sendo excluídos os que não atendessem aos critérios citados.

Os artigos foram avaliados, detalhadamente, seguindo os passos da análise temática de conteúdo de Bardin, respeitando as etapas metodológicas: pré-análise com a organização, leitura e escolha dos documentos. Em seguida a fase da exploração do material escolhido, uma etapa mais extensa que envolve o recorte, a enumeração e a classificação e, por fim, o tratamento dos dados que visa a in- terpretação para torná-los válidos. 5 Na pré-análise com a organização, foram encontrados 146 artigos.

Após a leitura dos títulos, foram selecionados 20 artigos, posteriormente a leitura dos resumos foram escolhidos 17 e, após a leitura na íntegra, foi excluído o que não atendeu a temática proposta, sendo selecionados por fim 16 artigos que atenderam aos critérios de inclusão (Tabela 1).

Durante a fase de exploração do material escolhido, através da leitura cuidadosa e criteriosa dos artigos, foi possível selecionar e identificar três categorias para abordagem: a) Violência moral, sexual e doméstica; b) Transtornos mentais comuns; c) Uso de álcool e drogas.

Tabela 1 - Resultados das buscas nas Bases de Dados, a partir dos descritores: Assistência à saúde”, "transtornos mentais" e "saúde da mulher" e seleção dos artigos

\begin{tabular}{|c|c|c|c|c|}
\hline \multirow[b]{2}{*}{ Base de Dados } & \multicolumn{4}{|c|}{ Artigos Selecionados } \\
\hline & Encontrados & $\begin{array}{l}\text { Leitura dos } \\
\text { Resumos }\end{array}$ & $\begin{array}{c}\text { Leitura dos } \\
\text { Títulos }\end{array}$ & $\begin{array}{l}\text { Leitura na } \\
\text { íntegra }\end{array}$ \\
\hline Lilacs & 24 & 10 & 8 & 8 \\
\hline Scielo & 12 & 6 & 8 & 7 \\
\hline Medline & 110 & 4 & 1 & 1 \\
\hline Total & 146 & 20 & 17 & 16 \\
\hline
\end{tabular}

\section{RESULTADOS E DICUSSÃO}

\section{- Violência Moral, Sexual e Doméstica}

A violência contra a mulher é um fato presente a um longo período na sociedade. Entretanto, essa problemática somente foi discutida como questão de saúde pública há pouco tempo, após a sanção da lei n 11.340/06, nomeada como Lei Maria da Penha, quando os casos de agressão física, sexual e moral começaram a ser questionados e refutados. ${ }^{6}$
A violência doméstica e familiar, dirigida a mulher, é compreendida como toda e qualquer forma de atitude ou conduta, fundamentada no sexo feminino, que gere dano ou sofrimento físico, sexual, psicológico, moral, patrimonial e morte, partindo de uma relação de convivência com a mulher, em estado presente, ou passado, independente de coabitação.7 
Através de um estudo, efetuado na cidade de Vitória no Espírito Santo em 2014, foi possível classificar a periodicidade dos principais tipos de violências contra a mulher e seu quantitativo, sendo a violência psicológica mais constante, com prevalência de $25,3 \%$, seguida pela violência física 9,9\% e, por fim, a violência sexual com $5,7 \%$. Identificou-se que, quanto aos fatores determinantes, a prevalência da violência está diretamente relacionada ao estado socioeconômico das mulheres, sendo a violência psicológica e física associada ao baixo nível de escolaridade, situação conjugal, histórico materno de violência por parceiro íntimo e uso de drogas. Em contrapartida, a violência sexual esteve associada apenas à renda familiar. ${ }^{8}$

No Brasil, os principais grupos de pessoas procuradas por vítimas de violência ao sofrerem uma agressão, são constituídos pelos familiares com $46 \%$, seguido por amigos $22,1 \%$, profissionais de saúde $13,4 \%$, educadores e religiosos 1,5\%. Essa procura se dá em maior número por vítimas do sexo feminino em comparação ao público masculino. 9

Na Espanha, nos serviços de atenção básica, constatou-se que mulheres que sofrem com abusos, são três vezes mais dispostas a desenvolverem sofrimentos psicológicos e queixas somáticas, e quatro vezes mais predispostas a usar medicações, quando comparadas a mulheres que não sofreram abusos. ${ }^{11}$

Apesar da violência física envolver agressões corporais, por ser mais perceptível, ainda apresenta índices inferiores em relação aos outros tipos de agressões, a exemplo do abuso psicológico que se torna o tipo de violência mais negligenciada. É caracterizada pela imposição do agressor quanto à liberdade das vítimas, além de restrições da autonomia individual, seguidas de repressão e humilhação. Entretanto, essa prática acaba passando despercebida entre os familiares e até mesmo entre os profissionais do serviço de saúde. 9

Frente a essa conjuntura, pode-se iden- tificar a necessidade de uma maior capacitação dos profissionais de saúde voltada a assistência à saúde mental, para atender as vítimas nas unidades, orientá-las e encaminhá-las para uma assistência integral, havendo uma intervenção adequada frente ao quadro de agressão presente. $^{11}$

Quando se refere a pessoas vítimas de violência, a assistência e o tratamento não se resumem ao paciente, mas também engloba toda a família e as pessoas evolvidas ao seu redor, pois o âmbito familiar funciona como forma de equilíbrio frente a diferentes situações e quando não existe a presença desse suporte, o processo de assistência torna-se dificultoso. ${ }^{12}$

\section{- Uso de álcool e outras drogas}

O consumo de drogas lícitas e ilícitas, durante a gestação, é considerado um problema de proporção social e de saúde pública, sendo um fator que sugestiona diretamente a adesão dessas mulheres ao pré-natal. São as grávidas com dependência química o público com menor participação nos grupos de gestantes e com maior probabilidade de desenvolver intercorrências obstétricas e fetais, além da dependência ter influência direta no estado social e emocional dessas mulheres. ${ }^{13}$

Decorrente de um estudo com 394 gestantes, realizado no município de Maringá, no estado do Paraná em 2013, foi constato que 72 mulheres (18,28\%), utilizavam alguma droga em abusão, significando que de cinco gestantes, uma usufruía de substâncias prejudiciais ao feto. Quanto a droga mais consumida pelas gestantes, o cigarro encontra-se em primeiro lugar e, consecutivamente, vem o álcool. Destaca-se que $1,52 \%$ das mulheres fizeram uso em conjunto as duas substâncias e 0,51\% usou a maconha, cocaína e álcool, concomitantemente. ${ }^{14}$

Casos de alcoolismo e o consumo de outras drogas são frequentemente atendidos nos serviços de atenção básica, estando geralmente 
associados a outros problemas e patologias. É dever dos profissionais do sistema de saúde reconhecer os sinais e sintomas referentes a esse quadro, orientar acerca das consequências clínicas, psicológicas e sociais que podem ser geradas a partir do uso dessas drogas em excesso. Alertar de que essa prática pode refletir nos familiares e pessoas ao seu redor, além de poder ser considerada uma sintomatologia para quadros de ansiedade ou depressão, sendo necessário o encaminhamento desse público ao serviço especializado da rede de atenção psicossocial, como o CAPS (Centro de Atenção Psicossocial). ${ }^{1}$

\section{- Transtornos Mentais Comuns}

Com a criação do Plano Nacional de Política para as Mulheres em 2013, a perspectiva da assistência quanto à saúde da mulher foi alterada, sendo agora priorizada a melhoria da qualidade de vida e promoção da saúde em todas as fases do ciclo de vida, levando em conta a área reprodutiva, física e psicológica. ${ }^{15}$

Em pesquisa, realizada na cidade de Ribeirão Preto (SP), foi evidenciada a correlação entre as manifestações de Transtornos Mentais Comuns (TMC) e o sexo feminino, sendo o transtorno um fator influenciador dos padrões somáticos. Devido ao grande fluxo de mulheres usuárias de unidades de saúde, há uma maior prevalência do uso de psicofármacos por esse público, principalmente para o tratamento de depressão, ansiedade e enxaqueca. No entanto, a maioria dessas mulheres não fazem uso de forma adequada, colocando assim, a sua saúde em risco. ${ }^{16}$

A vida conjugal conturbada, gravidez com ou sem a presença de um parceiro, ter dois ou mais filhos, não ter ocupação com vínculo empregatício, nível socioeconômico baixo, baixa escolaridade, são fatores que aumentam o risco de apresentar um diagnóstico de transtornos mentais. Simultaneamente, a enxaqueca e o estresse nas fases de maior prevalência dos sintomas, aumentam o risco de desenvolver quadros de ansiedades e depressão, intensificando a predisposição para desencadear os TMC. ${ }^{17}$

O uso de uma assistência diferenciada proporciona um impacto significativo no quadro psicossocial desse público, ao conceder um momento de reflexão e crítica a essas mulheres, possibilitando que se expressem, para relatar suas angústias, bem como uma abertura para entender o meio onde elas vivem, além de introduzi-las na promoção de serviço de saúde mental. ${ }^{19}$

\section{CONSIDERAÇÕES FINAIS}

Após a leitura dos artigos selecionados, foi possível identificar os principais motivos que levam as mulheres a buscarem os serviços de atenção primária, o contexto em que estão inseridas, bem como o comportamento exercido pelos profissionais presentes na assistência. Analisando a conjuntura da população femi- nina, percebeu-se que as mulheres em sofrimento mental têm inúmeras razões que interferem em seu estado de saúde, estando a violência e o abuso no consumo de álcool e outras drogas os fatores mais relacionados a etiologia dos transtornos mentais, além de ser uma das principais causas da procura pelos serviços de saúde. 
Outro fator importante é o déficit de conhecimento dos profissionais que realizam o atendimento a esse público. Constatou-se a necessidade de uma maior capacitação e preparação, para torná-los aptos a prestarem uma assistência de qualidade, humanizada e integral, utilizando a escuta qualificada e garantindo a continuidade do cuidado.

\title{
MENTAL HEALTH CARE FOR WOMEN IN THE PRIMARY CARE UNIT
}

\section{ABSTRACT}

Basic care is classified as the main form of access of the user to the Health System Units, providing collective and individual actions, aimed at prevention, promotion and protection of health, as well as early diagnosis, adherence to treatment and rehabilitation, promoting improvement well-being of collectivities. The objective of this study was to review the literature on the presence of the female population in the primary health care service, which comes from complaints related to mental health. This is a bibliographical survey, carried out from 2011 to 2019, in the databases Lilacs, Scielo and Medline. The descriptors Health care, mental disorders and women's health were used. Exclusion criteria were adopted: articles published before 2011, which had no methodological consistency and were not related to the theme. Twenty articles were analyzed and only 16 after preliminary reading met the inclusion criteria. Data analysis was performed through the thematic content analysis, proposed by Bardin. Through the careful reading of the articles, three categories were identified: moral, sexual and domestic violence, mental disorders more prevalent and use of alcohol and other drugs. It was evidenced that psychological violence has the highest prevalence, followed by physical violence, having as determinants the low level of schooling, marital status, maternal history of intimate partner violence and drug use, and finally, sexual violence, as the third most prevalent type associated with family income, predisposing the development of common mental disorders, as well as abusive use of psychotropic drugs, stress, anxiety and marital problems. In this sense, it is understood that women in mental suffering have many reasons that trigger their state of health, being to violence and abuse in the consumption of alcohol and other drugs, among the factors most related to the etiology of mental disorders.
\end{abstract}

KEYWORDS: Health Care, Mental Disorders, Women's Health.

\section{REFERÊNCIAS}

1. Brasil. Ministério da Saúde. Saúde Mental Cadernos de Atenção Básica, $n^{\circ}$ 34. [online] Brasília (DF); 2013. Disponível em: http://dab.saude.gov. br/portaldab/biblioteca.php?conteudo=publicacoes/cab34.
2. Arce VAR, Sousa, Lima MG. A práxis da Saúde Mental no âmbito da Estratégia Saúde da Família: contribuições para a construção de um cuidado integrado. Physis: Rev. de Saúde Coletiva. Rio de Janeiro Abril. 2011; 21(2): 541-60. 
3. Borges TL, Hegadoren KM, Miasso Al. Transtornos mentais comuns e uso de psicofármacosem mulheres atendidas em unidades básicas de saúde em um centro urbano brasileiro. Rev Panam Salud Publica. 2015; 38(3): 195-201.

4. Gonçalves AMC, Teixeira MTB, Gama JRA, Lopes $C S$, Silva GA, Gamarra $C$, et al. Prevalência de depressão e fatores associados em mulheres atendidas pela Estratégia de Saúde da Família. Jornal Brasileiro Psiquiatr. Janeiro 2018; 67(2): 101-9.

5. Bardin L. Análise de conteúdo. São Paulo: Edições 70; 2016.

6. Brasil. Diário Oficial da União. Lei n. 11.340, de 7 de agosto de 2006. Dispõe sobre a violência doméstica contra a mulher [online]. Brasília (DF); 2006. Disponível em: http://www.planalto.gov. br/ccivil_03/_ato2004-2006/2006/lei//11340.htm.

7. Santos WJ, Oliveira PP, Viegas SMF, Ramos TM, Policarpo AG, Silveira EAA. Violência Doméstica Contra a Mulher Perpetrada por Parceiro Íntimo: Representações Sociais de Profissionais da Atenção Primária à Saúde. Rev. Onl. Peq. Cuid. Fundam. Jul/Set. 2018; 10(3): 770-77.

8. Leite FMC, Amorim MHC, Wehrmeister FC, Gigante DP. Violência contra a mulher em Vitória, Espírito Santo, Brasil. Rev. Saúde Pública. [online] São Paulo 2017; 51(10).

9. Soares JSF, Lopes MJM, Njaine K. Violência nos relacionamentos afetivo-sexuais entre adolescentes de Porto Alegre, Rio Grande do Sul, Brasil: busca de ajuda e rede de apoio. Cad. Saúde Pública. Rio de Janeiro Jun. 2013; 29(6): 112730.

10. Martín-Baenaa D, Montero-Piñara I, Escribà-Agüira V,Vives-Casesa C. Violence against young
VOLUME 17 - NÚMERO 2 - AGO/2019

ISSN ELETRÔNICO 2317-7160

women attending primary care services in Spain: prevalence and health consequences. Family Practice. [online] 2015; 32(4):381-86.

11. Gomesa NP, Erdmannb AL, Stulpb KP, Diniz NMF, Correia CM, Andrade SR. Cuidado às muIheres em situação de violência conjugal importância do psicólogo na Estratégia de Saúde da Família. Psicol. USP. São Paulo Jan/Apr. 2014; 25(1): 63-9.

12. Hermel SJ, Drehmer LBR. Repercussões da violência intrafamiliar: Um estudo com mulheres em acompanhamento psicológico. Psicol. Argum. Curitiba Jul/Set. 2013; 31(74), 437-46.

13. Kassada DS, Marcon SS, Pagliarini WMAP. Percepções e práticas de gestantes atendidas na atenção primária frente ao uso de drogas. Esc Anna Nery. Set. 2014; 18(3): 428-34.

14. Kassada DS, Marcon SS, Pagliarini MA, Rossi RM. Prevalência do uso de drogas de abuso por gestantes. Acta Paul. Enferm. São Paulo 2013; 26(5): 467-71.

15. Pasquala KK, Carvalhaes MABL, Paradac CMGL Atenção à saúde da mulher após os 50 anos: vulnerabilidade programática na Estratégia Saúde da Família. Rev Gaúcha Enferm. Porto Alegre, Jun. 2015; 36(2): 21-7.

16. Moliner J, Lopes SMB. Saúde mental na atenção básica: possibilidades para uma prática voltada para a ampliação e integralidade da saúde mental. Saúde Soc. São Paulo 2013; 22(4): 10721083.

17. Almeida SM, Nunes MA, Camey S, Pinheiro AP, Schmidt MI. Transtornos mentais em uma amostra de gestantes da rede de atenção básica de saúde no Sul do Brasil. Cad. Saúde Pública. 2012; 28(2):385-394. 
revista de NOVA
ciências

daiênaúde ESPERANÇA

18. Correia LL, Linhares MBM. Enxaqueca e Estresse em Mulheres no Contexto da Atenção Primária. Psicologia: Teoria e Pesquisa. Abr-Jun. 2014; 30(2): 145-152.
19. Cavalcante AC, Silva RM. Experiências psíquicas de mulheres frequentadoras da rede pública de saúde em Teresina (PI, Brasil). Ciência \& Saúde Coletiva. Rio de Janeiro 2011; 16(4): 2211-20. 